\title{
Frustration-induced anomalous transport and strong photon decay in waveguide QED
}

\author{
Ron Belyansky $\odot,{ }^{1,2, *}$ Seth Whitsitt, ${ }^{1,2}$ Rex Lundgren,,${ }^{1,2}$ Yidan Wang, ${ }^{2}$ Andrei Vrajitoarea,,${ }^{3, \dagger}$ \\ Andrew A. Houck, ${ }^{3}$ and Alexey V. Gorshkov $\circledast^{1,2}$ \\ ${ }^{1}$ Joint Center for Quantum Information and Computer Science, NIST/University of Maryland, College Park, Maryland 20742, USA \\ ${ }^{2}$ Joint Quantum Institute, NIST/University of Maryland, College Park, Maryland 20742, USA \\ ${ }^{3}$ Department of Electrical Engineering, Princeton University, Princeton, New Jersey 08540, USA
}

(Received 24 July 2020; accepted 16 August 2021; published 7 September 2021)

\begin{abstract}
We study the propagation of photons in a one-dimensional environment consisting of two noninteracting species of photons frustratingly coupled to a single spin $1 / 2$. The ultrastrong frustrated coupling leads to an extreme mixing of the light and matter degrees of freedom, resulting in the disintegration of the spin and a breakdown of the "dressed-spin," or polaron, description. Using a combination of numerical and analytical methods, we show that the elastic response becomes increasingly weak at the effective spin frequency, showing instead an increasingly strong and broadband response at higher energies. We also show that the photons can decay into multiple photons of smaller energies. The total probability of these inelastic processes can be as large as the total elastic scattering rate, or half of the total scattering rate, which is as large as it can be. The frustrated spin induces strong anisotropic photon-photon interactions that are dominated by interspecies interactions. Our results are relevant to state-of-the-art circuit and cavity quantum electrodynamics experiments.
\end{abstract}

DOI: 10.1103/PhysRevResearch.3.L032058

Introduction. Photons propagating in one-dimensional environments are a fundamental building block for quantum optics and waveguide quantum electrodynamics (QED). While interaction among photons is inherently negligible, strong effective interactions can be induced by coupling the light to atoms, or "impurities." Such photon-photon interactions are a crucial ingredient in many technologies ranging from quantum communication to quantum computation and metrology [1-4]. Even a single two-level-atom (or a spin-1/2) can induce nontrivial behavior, perfectly reflecting photons whose energy matches the two-level gap $\Delta$, while being transparent for other photons [5-8].

This picture can be greatly modified when the lightmatter coupling is increased to the so-called ultrastrong coupling (USC) regime of waveguide QED [9-14]. This regime has been recently of great experimental and theoretical interest $[2-4,15,16]$, and has been experimentally realized in superconducting quantum circuits [17-21], allowing the exploration of quantum many-body physics with a single artificial atom [22]. The hallmark feature of the USC regime is the breakdown of the rotating-wave approximation and the description of light and matter as separate entities, which must instead be described by hybridized excitations.

\footnotetext{
*rbelyans@umd.edu

†Present address: James Franck Institute and Department of Physics, University of Chicago, Chicago, Illinois 60637, USA.

Published by the American Physical Society under the terms of the Creative Commons Attribution 4.0 International license. Further distribution of this work must maintain attribution to the author(s) and the published article's title, journal citation, and DOI.
}

Nevertheless, most light-matter systems do admit an intuitive interpretation in terms of quasiparticles whose behavior closely resembles the bare constituents of the system. For a two-level atom coupled to a 1D continuum, such a hybridized description is given in terms of a "dressed spin" or a polaron [23-25]. The strong dressing of the spin by photons leads to a dramatic Lamb shift of the bare spin frequency $\Delta$ to a renormalized value $\Delta_{R} \ll \Delta$ [26], the energy of the polaron excitation. The propagation of photons in the system can be understood in terms of scattering of free photons off the polaron, with the scattering resonance being shifted from $\Delta$ to $\Delta_{R}[10,14]$. This renormalized frequency emerges as the natural intrinsic energy scale of the system, with all nontrivial physics, such as photon-photon interactions, occurring in the vicinity of $\Delta_{R}$. This intuition can be formalized with the wellknown variational polaron transformation, which has been widely successful in describing both static and dynamical observables in various spin-boson systems [14,27-34].

In this Letter, we introduce a regime of light-matter interaction where the dressed-spin quasiparticle description of the combined light-matter system qualitatively breaks down. This is induced by ultrastrong frustrated interactions between a single two-level atom and two different species of photons in one dimension [35,36] (see Fig. 1). We use matrix-product-state (MPS) numerics together with field-theoretical calculations to study the propagation of a single photon in the system. At weaker couplings, the elastic scattering shows a peaked response at a renormalized value $\Delta_{R}<\Delta$, consistent with the polaron interpretation. However, at larger couplings, this resonance becomes increasingly weak, and instead there is an emergent increasingly large and broadband response at large frequencies $\omega>\Delta_{R}$. We also find that inelastic processes, where the photon decays into several smaller-energies photons, can be as important or even dominate the elastic 


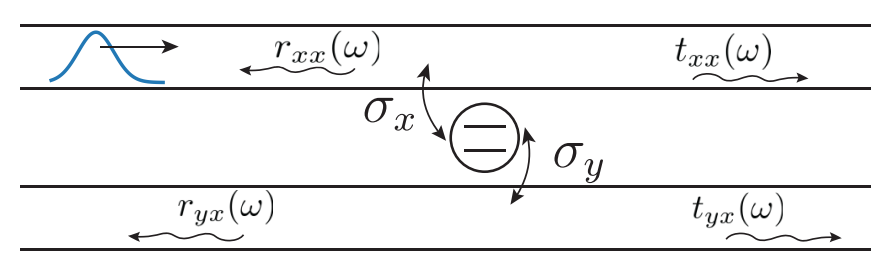

FIG. 1. Schematic of the model, where a single spin $1 / 2$ is coupled locally to two independent electromagnetic fields, represented here as two separate waveguides.

scattering. This decay rate does not peak in the vicinity of $\Delta_{R}$, in contrast to the polaron scenario, but saturates close to its allowed maximum and persists at very high energies, exceeding even the bare gap $\Delta$. Both the elastic and inelastic results show that the induced photon-photon interactions can be highly anisotropic, being dominated by interactions between photons of different species.

The model we study is closely related to the problem of a spin coupled to two competing Ohmic baths. The groundstate phase diagram and the spin properties in such a system were originally studied in the context of quantum impurities in magnetically ordered backgrounds [35-40], where it was observed that the two competing baths result in the preservation of coherence in the spin dynamics, which was named "quantum frustration of decoherence" [35,36]. Here we are instead interested in the dynamics of the photons.

Model. We consider a single spin- $1 / 2$ that is coupled, via two noncommuting operators, to two species of propagating photons in one dimension, as shown in Fig. 1, and as described by the Hamiltonian $[35,36](\hbar=1)$

$$
\begin{aligned}
\hat{\mathcal{H}}= & \sum_{i=x, y} \int d z \frac{1}{2}\left\{q_{i}(z)^{2}+\left[\partial_{z} \phi_{i}(z)\right]^{2}\right\}-\frac{\Delta}{2} \hat{\sigma}_{z} \\
& +\pi \sqrt{\alpha_{x}} q_{x}(0) \hat{\sigma}_{x}+\pi \sqrt{\alpha_{y}} \partial_{z} \phi_{y}(0) \hat{\sigma}_{y} .
\end{aligned}
$$

The two photon species have a linear dispersion $\omega_{k}=$ $|k|$ and are described by the scalar fields $\phi_{i}(z)$ satisfying $\left[q_{i}(z), \phi_{j}\left(z^{\prime}\right)\right]=-i \delta_{i j} \delta\left(z-z^{\prime}\right)$. Here, $q_{i}(z)$ and $\phi_{i}(z)$ could represent the charge and flux degrees of freedom of two superconducting transmission lines [10], and the spin degree of freedom can be a qubit that is coupled capacitively to one transmission line and inductively to the other [41]. We note that our results would apply equally well to other geometries, such as a spin coupled to two semi-infinite leads [11], or a spin coupled to two polarizations of a single waveguide as in Ref. [42]. In Eq. (1), $\alpha_{i}(i=x, y)$ are the dimensionless coupling constants, which, for the rest of the Letter, we assume to be equal $\left(\alpha_{x}=\alpha_{y} \equiv \alpha\right)$.

The Hamiltonian in Eq. (1) needs to be supplemented with an ultraviolet cutoff $\omega_{c}$. The latter can be used to define, via a renormalization group (RG) procedure [36], a renormalized spin frequency $\Delta_{R}$, implicitly given by

$$
\Delta_{R}=\frac{\Delta}{1+2 \alpha \ln \left(\omega_{c} / \Delta_{R}\right)} .
$$

This quantity, derived in Refs. $[35,36]$, is close to the bare spin frequency $\Delta$ for small $\alpha \rightarrow 0$, and it decreases as $\alpha$ is increased, approaching 0 as $\alpha \rightarrow \infty$. As we show in the next section, for intermediate coupling strengths $\alpha \lesssim 0.4, \Delta_{R}$ plays an important role in the photon dynamics, where it can be interpreted as the splitting of the dressed spin, whereas this picture breaks down for larger $\alpha$ (see Fig. 2).

Anomalous transport. We begin by considering the elastic scattering of a single photon. Without loss of generality, we assume an incoming $x$ photon that can scatter elastically in four different ways, as shown in Fig. 1. We computed the scattering coefficients both numerically, using an MPS-based approach, and analytically, with diagrammatic perturbation theory. In order to simulate the system numerically, we use an orthogonal polynomials mapping $[43,44]$ that transforms Eq. (1) into a one-dimensional tight-binding model with only local interactions [see the Supplemental Material (SM) [45]]. We first use the density matrix renormalization group method to find the ground state of the system and then create a broad-in-frequency single-photon wave packet on top of it. This state is then evolved for sufficiently long times so that the scattering process has ended. From the resulting state, we extract the elastic probabilities [46], shown in the top row of Fig. 2, as a function of the incoming frequency $\omega$ and coupling constant $\alpha$.

In order to gain analytical insight into the problem, we use the fact that the elastic $S$ matrix can be written in terms of spin susceptibilities $[11,12,18,47-50]$. For the setup in Fig. 1, we find [45]

$$
r_{\alpha \beta}(\omega)=-i 2 \pi \alpha \omega \chi_{\alpha \beta}(\omega), \quad t_{\alpha \beta}(\omega)=\delta_{\alpha \beta}+r_{\alpha \beta}(\omega),
$$

where the spin susceptibilities $\chi_{\alpha \beta}(\omega)$ are given by the Fourier-transformed retarded Green's function

$$
\chi_{\alpha \beta}(\omega)=-\frac{i}{4} \int_{0}^{\infty} d t e^{i \omega t}\left\langle\left[\hat{\sigma}_{\alpha}(t), \hat{\sigma}_{\beta}(0)\right]\right\rangle,
$$

evaluated in the ground state. Equations (3) and (4) are exact for a single incoming photon, but they can be understood intuitively within linear response formalism. The scattering of a $\beta$ photon acts as a perturbation $\hat{\sigma}_{\beta}(0)$ on the spin, and the response $\hat{\sigma}_{\alpha}(t)$ of the spin describes the emission of an $\alpha$ photon.

The advantage of writing the elastic $S$ matrix in the form of Eqs. (3) and (4) is that it allows the use of powerful field-theoretical methods. In particular, we use an Abrikosov pseudofermion representation of the spin to perturbatively compute Eq. (4) to leading order in $\alpha$, and employ the Dyson equation to sum an infinite subset of diagrams, as in the random-phase approximation of the Coulomb gas [51]. We then use the Callan-Symanzik equation together with the RG flow equations from Refs. [35,36] to improve upon the perturbative results, taking into account the nonperturbative Lamb shift in Eq. (2). The end result is (see the SM for derivation [45])

$$
\begin{aligned}
& \chi_{x x}(\omega)=\frac{(-\Delta+i \pi \alpha \omega) / 2}{\Delta^{2}-\omega^{2}\left[\pi^{2} \alpha^{2}+\left[1+2 \alpha \ln \left(\frac{\omega_{c}}{\omega}\right)\right]^{2}\right]-i 2 \pi \alpha \Delta \omega}, \\
& \chi_{x y}(\omega)=\frac{-i \omega\left[1+2 \alpha \ln \left(\omega_{c} / \omega\right)\right] / 2}{\Delta^{2}-\omega^{2}\left[\pi^{2} \alpha^{2}+\left[1+2 \alpha \ln \left(\frac{\omega_{c}}{\omega}\right)\right]^{2}\right]-i 2 \pi \alpha \Delta \omega} .
\end{aligned}
$$

These forms for the susceptibility have a peak near $\Delta_{R}$ with a width of order $\tau^{-1} \sim \alpha \Delta_{R}$, where $\tau$ is the lifetime of a 


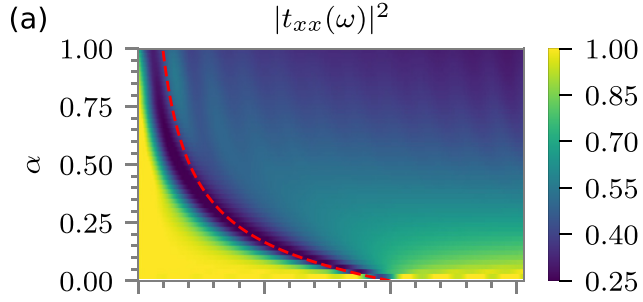

(d)

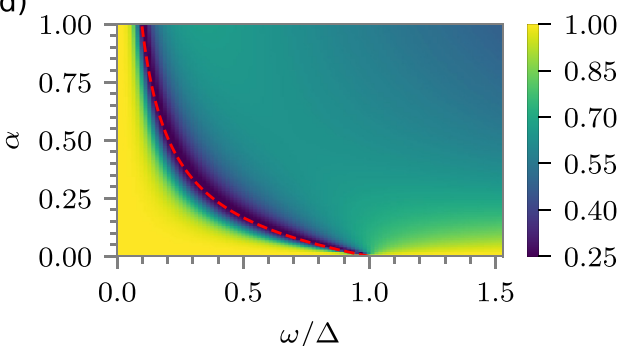

(b)

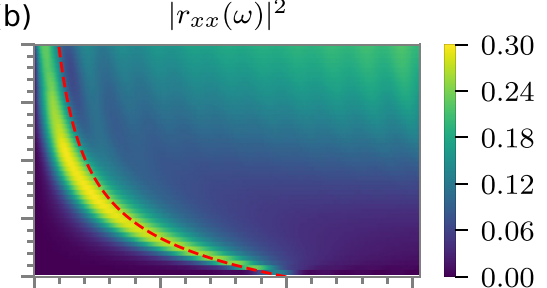

(e)

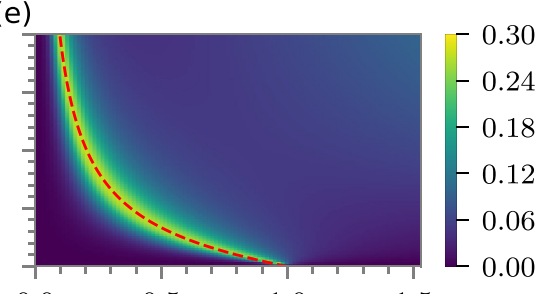

0.5 $\omega / \Delta$

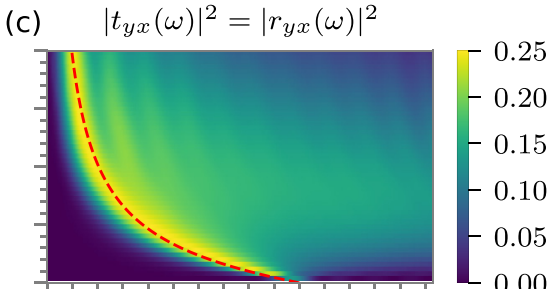

(f)

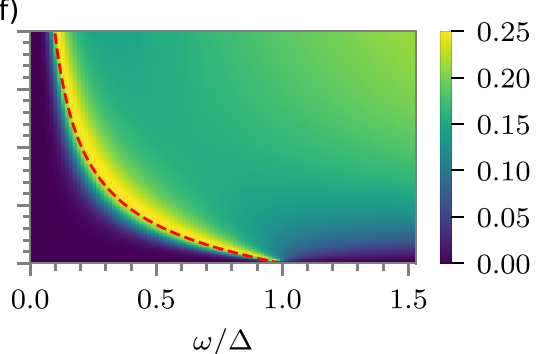

FIG. 2. Numerical (a)-(c) and analytical (d)-(f) elastic scattering coefficients corresponding to Fig. 1, as a function of the incoming frequency $\omega$ and coupling constant $\alpha$. The red dashed line corresponds to $\Delta_{R}$ from Eq. (2). The cutoff is $\omega_{c}=10 \Delta$. The oscillating behavior in the numerical plots at large $\alpha$ is a finite-size effect due to the scattering being very broad in space-time.

spin excitation. At small $\alpha$, both expressions reduce to narrow peaks at $\Delta$, since $\Delta_{R} \rightarrow \Delta$ and $\tau^{-1} \rightarrow 0$ for $\alpha \rightarrow 0$. The resulting transmission and reflection probabilities are shown in the bottom row of Fig. 2.

We find excellent qualitative agreement between the numerical and analytical results, particularly for $\alpha \lesssim 0.5$. At very small $\alpha$, we have the standard situation in waveguide QED [5-8], where only photons at $\omega \approx \Delta$ are coupled to the spin and experience scattering, being equally split among the four channels in Fig. 1, and the rest are simply transmitted. As $\alpha$ is increased, the location of the resonance drastically decreases, in excellent agreement with the RG predicted $\Delta_{R}$ in Eq. (2) (red dashed lines in Fig. 2).

For $\omega \ll \Delta_{R}$, Fig. 2 shows perfect transmission for all $\alpha$, indicating that modes with frequencies smaller than $\Delta_{R}$ are effectively uncoupled from the impurity. This regime is qualitatively similar to that of the usual unfrustrated spin-boson model [26] and the Kondo problem [52]. In the latter, for energies smaller than the Kondo temperature (the equivalent of $\Delta_{R}$ ), the impurity is screened and essentially disappears from the problem [50,52,53].

The $\omega>\Delta_{R}$ regime, on the other hand, is drastically different than in these paradigmatic models and the standard ultrastrong waveguide QED systems (see the SM for a more detailed comparison to the case when the coupling operator to both waveguides is the same [45]). Surprisingly, we find that, at large $\alpha$, there is very little transmission, even for $\omega \gg \Delta_{R}$. For $\alpha \lesssim 0.4$, the system still admits the effective polaron description, since the strongest elastic response for all scattering channels in Fig. 2 is still concentrated near $\Delta_{R}$. This picture changes dramatically for $\alpha \gtrsim 0.4$, where the reflection $\left|r_{x x}(\omega)\right|^{2}$, for example, instead of monotonically decreasing away from the resonance at $\Delta_{R}$, first decreases but then starts increasing for $\omega>\Delta_{R}$. This behavior is more easily seen in the numerical plots but is nonetheless qualitatively consistent with the analytical solution. In particular, from Eq. (5) we see that, at large $\alpha$ and $\omega \gg \Delta_{R}, \chi_{x x}(\omega)$ decays sublinearly $\sim \omega^{-1} \ln ^{-2}\left(\omega_{c} / \omega\right)$, as was also pointed out in Refs. [35,36]. Hence, the reflection coefficient $\left[\sim \omega \chi_{x x}(\omega)\right.$ from Eq. (3)] increases, while the transmission decreases, in that regime. At even higher couplings $\alpha \gtrsim 0.5$, the numerical results show that the $\Delta_{R}$ resonance in $\left|\widetilde{r_{x x}}(\omega)\right|^{2}$ becomes increasingly weaker, becoming less intense than the extremely broadband response at higher frequencies. All this implies that the spectral weight of the $\operatorname{spin}\left\{\sim \operatorname{Im}\left[\chi_{x x}(\omega)\right]\right\}$ becomes increasingly spread out over larger energies instead of having a sharp peak at $\Delta_{R}$. This anomalous behavior of the elastic reflection and transmission at large $\alpha$, bearing no resemblance to a two-level system, constitutes the first of the two main results of this work.

Another interesting aspect in Fig. 2 is the behavior of the interspecies scattering, $\left|t_{y x}(\omega)\right|^{2}$, where the $\Delta_{R}$ resonance becomes extremely broad on the $\omega>\Delta_{R}$ side (note that $\chi_{x y}(\omega)$ [Eq. (6)] approaches a constant for large $\alpha$ and $\omega \gg \Delta_{R}$ ). This implies that the incoming $x$ photon can be efficiently converted into a $y$ photon in a wide range of energies. The interspecies scattering at large $\alpha$ shows significant disagreement between the numerical and analytical results, with the analytics suggesting that $\left|t_{y x}(\omega)\right|^{2}$ increases as $\omega$ is increased away from the $\Delta_{R}$ resonance. The numerics do not show such an increase, but rather show that $\left|t_{y x}(\omega)\right|^{2}$ approaches zero for very large $\omega$ and $\alpha$. As we discuss in the next section, the discrepancy in $\left|t_{y x}(\omega)\right|^{2}$ [as well as in $\left|r_{x x}(\omega)\right|^{2}$ and $\left|t_{x x}(\omega)\right|^{2}$ ] at large $\alpha$ is due to the lack of certain kind of $\mathcal{O}\left(\alpha^{2}\right)$ diagrams in the susceptibility calculation and is related to the presence of substantial inelastic scattering.

Photon decay. As is well known, ultrastrong coupling can give rise to number-nonconserving inelastic processes. The probability of such processes is, however, typically much weaker than the elastic rate and is usually peaked at the vicinity of the polaron energy $\Delta_{R}[11,48,50]$. As we now show, these two expectations are strongly invalidated due to the strong frustration in our model, which constitutes the second main unexpected result of this work.

Conservation of probability implies that $\left|t_{x x}(\omega)\right|^{2}+$ $\left|r_{x x}(\omega)\right|^{2}+2\left|t_{y x}(\omega)\right|^{2}=1-\gamma(\omega)$, where nonzero $\gamma(\omega)$ sig- 

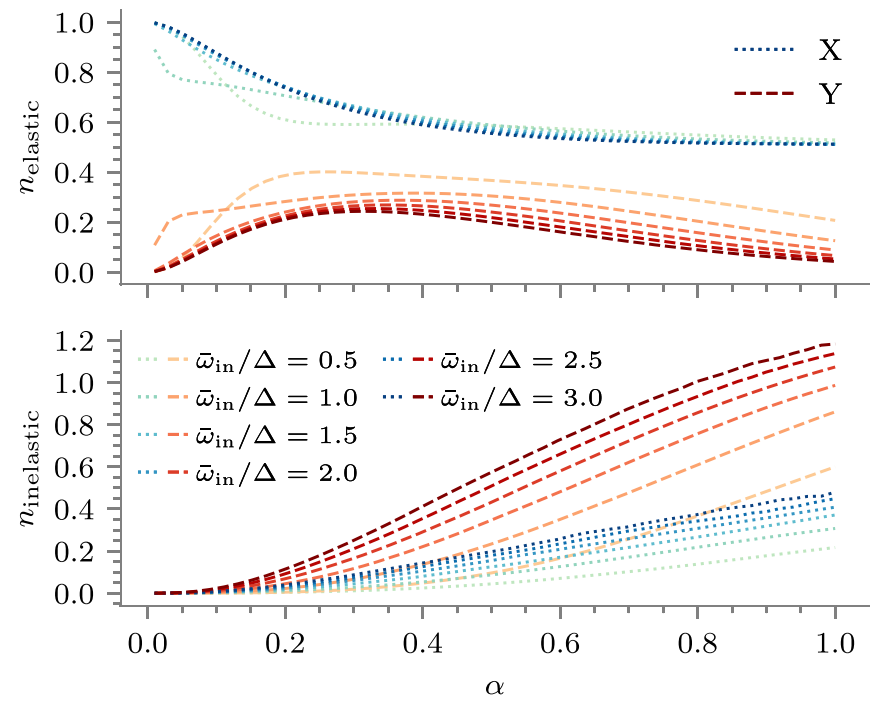

FIG. 3. Numerically computed total number of elastic (a) and inelastic (b) particles produced in each waveguide as a function of $\alpha$ for six different incoming wave packets. The wave packets are single-particle Gaussians centered at $\bar{\omega}_{i n}$ with a standard deviation of $0.2 \Delta . \omega_{c}=10 \Delta$.

nifies that the initial $x$ photon of energy $\omega$ can decay into multiple photons of smaller energies. Direct computation using Eqs. (3), (5), and (6) yields $\gamma(\omega)=0$, which is certainly incorrect. In fact, the numerical plots in Fig. 2 show that the total inelastic scattering rate approaches $\approx 0.5$ (for $\omega \gtrsim 0.5 \Delta$ and $\alpha \gtrsim 0.6$ ). In those regimes, a photon is therefore as likely to decay as to be scattered elastically. Note that the continuity equation in Eq. (3) constrains that $\max [\gamma]=0.5$, implying that the scattering is nearly maximally inelastic in that regime.

To get a deeper understanding of the inelastic scattering, we perform additional numerical simulations and analytical computations. Numerically, we use narrower wave packets in order to probe the dependence of the outgoing particles on the energy of the incoming photon. After the scattering event, we record the total number of elastically and inelastically scattered photons in each waveguide [45], shown in Fig. 3 for six wave packets with different mean energy. The first observation from Fig. 3 is that the inelastic emission is highly anisotropic, giving rise to significantly more $y$ photons than $x$ photons, for an initial $x$ wave packet. Since the scattering process cannot change the state of the spin, the leading-order inelastic process involves four (one incoming and three outgoing) photons and is therefore of order $\alpha^{2}$ [11]. It is precisely those diagrams which are missing in the susceptibilities in Eqs. (5) and (6), explaining also why the analytics become inaccurate for $\alpha \gtrsim 0.5$ where nearly half of the scattering is inelastic. The four leading-order inelastic processes are $x \rightarrow\{x x x, y y y, x x y, x y y\}$. We denote the probability of these processes by $\gamma_{\mu_{1} \mu_{2} \mu_{3}}\left(\omega_{1}, \omega_{2}, \omega_{3} ; \omega\right)$ where $\mu_{i}$ specifies the flavor of the outgoing photon $\left(x\right.$ or $y$ ) and $\omega_{i}$ its frequency. Energy conservation constrains $\omega_{1}+\omega_{2}+\omega_{3}=$ $\omega$. We have computed the leading order diagrams contributing to these processes, and the expressions are provided in the SM [45].

We find that the leading-order expression for $\gamma_{x x x}$ exactly matches that [11] of the standard unfrustrated spin-boson model. Moreover, three of the four processes are elegantly related to each other to leading order, as follows:

$$
\gamma_{y y y}=\gamma_{x x x} \frac{\omega^{2}}{\Delta_{R}^{2}}, \quad \gamma_{x x y}=\gamma_{x x x} \frac{\omega_{3}^{2}}{\Delta_{R}^{2}} .
$$

The first of these demonstrates that an incoming $x$ photon with energy $\omega>\Delta_{R}$ is more likely to decay into three $y$ photons, as opposed to three $x$ photons. The second relation shows that the $\gamma_{x x y}$ process is more likely to occur than $\gamma_{x x x}$ provided that the energy of the $y$ photon satisfies $\omega_{3}>\Delta_{R}$. However, it is far less likely compared to $\gamma_{y y y}$ because if $\omega_{3} \approx \omega$, energy conservation would require $\omega_{1} \approx \omega_{2} \approx 0$ and this would highly suppress its probability. The remaining process, $\gamma_{x y y}$, does not have a simple relation to the other three, but we have verified by direct numerical integration that its total cross section is of the same order as the one for $\gamma_{x x y}$, and both of these are significantly less important than $\gamma_{y y y}$. In short, all this demonstrates that, in the regime $\omega \gg \Delta_{R}$, photons of one flavor decay dominantly to the other. This agrees qualitatively with Fig. 3, since even the smallest energy wave packet $\left(\bar{\omega}_{i n} / \Delta=0.5\right)$ is in the regime of $\omega>\Delta_{R}$ for $\alpha \gtrsim 0.2$ (see Fig. 2). In fact, for almost all the wave packets and the range of $\alpha$ in Fig. 3, we have $\omega \gg \Delta_{R}$.

Figure 3 also shows that the number of elastic $y$ photons goes to zero at large $\alpha$ and $\omega$, consistent with $t_{y x}(\omega) \rightarrow 0$ in that regime, as we discussed in the previous section. Interestingly, this says that the interspecies scattering can be completely inelastic, while also dominating over the intraspecies scattering, as we have just shown. Remarkably, we also see that the number of inelastically produced photons continues to rise as a function of $\bar{\omega}_{\text {in }}$, suggesting that $\gamma(\omega)$ remains close to 0.5 even for $\omega>\Delta \gg \Delta_{R}$. This behavior is consistent with the anomalous elastic scattering we identified in the previous section, but we nonetheless expect that $\gamma(\omega)$, as well as the nontrivial elastic scattering $\left[r_{x x}(\omega), t_{y x}(\omega)\right]$, would eventually decay to zero as $\omega \rightarrow \infty$. While all the presented results are qualitatively independent of the high-energy cutoff, we conjecture that the exact location of this decay may be nonuniversal and may depend on the precise cutoff function for a given physical system. In the SM [45], we compare these results to the situation where the two waveguides in Fig. 1 couple to the spin via the same operator $\hat{\sigma}_{x}$, showing that, without the frustrated coupling, the inelastic processes are comparatively negligible and the anomalous elastic transport is absent.

Summary and outlook. In this work we have shown that ultrastrong frustrated coupling between a two-level system and free photons in one dimension leads to anomalous photon transport and maximal photon decay. This behavior bears no resemblance to scattering off a two-level system and hence indicates the breakdown of the polaron quasiparticle description. Instead, this is reminiscent of non-Fermi-liquid behavior of quantum impurity models in strongly correlated electron systems $[48,50]$.

While in this Letter we have focused solely on the equal couplings case $\alpha_{x}=\alpha_{y}$, we expect our main results, namely the anomalous transport and strong photon decay, to remain qualitatively valid even in the presence of anisotropic couplings, provided they are both large and similar in magnitude. On the other hand, if the couplings are strongly asymmetric, 
say $\alpha_{x} \gg \alpha_{y}$, the behavior of the system would resemble more the unfrustrated model. The weaker coupling in such a case can be thought of as an unwanted source of dissipation acting on the spin, which would necessarily be present experimentally. Such unwanted dissipation can be similarly included in our model by adding a third waveguide with coupling $\alpha_{3} \ll \alpha_{x} \approx \alpha_{y}$. In superconducting circuits, such additional dissipation channels can be made negligible compared to the desired couplings [17,18], and thus should not qualitatively affect our results.

Future theoretical work can investigate what kind of effective spin-spin interactions as well as novel phases of hybrid light-matter systems can be engineered by adding multiple impurities. The numerical and analytical methods developed in this work can also be immediately applied in a variety of other situations, such as photons with more exotic dispersions. It would also be interesting to develop protocols that make use of the unusual properties of the light-matter system in this work for entanglement generation, single-photon switches and routers, and frequency conversion, among other applica- tions. Finally, our work may also shed light and inspire future studies on the problems of heat and energy transport, relevant for quantum thermodynamics and quantum chemistry, where similar models to the one studied here appear [54,55].

Acknowledgments. We acknowledge valuable discussions with P. Bienias, M. Plenio, and I. Boettcher. We thank P. Bienias for comments on the manuscript. R.B., R.L., Y.W., and A.V.G. acknowledge funding by ARO MURI, U.S. Department of Energy Award No. DE-SC0019449, NSF PFC at JQI, DoE ASCR Quantum Testbed Pathfinder program (Award No. DE-SC0019040), DoE ASCR Accelerated Research in Quantum Computing program (Award No. DESC0020312), NSF PFCQC program, AFOSR, ARL CDQI, and AFOSR MURI. A.V. and A.A.H. acknowledge funding by NSF (PHY-1607160). R.B. acknowledges support of NSERC and FRQNT of Canada. The authors acknowledge the University of Maryland supercomputing resources [56] made available for conducting the research reported in this Letter. Numerical simulations were performed using the ITensor Library [57].
[1] D. Roy, C. M. Wilson, and O. Firstenberg, Colloquium: Strongly interacting photons in one-dimensional continuum, Rev. Mod. Phys. 89, 021001 (2017).

[2] A. Frisk Kockum, A. Miranowicz, S. De Liberato, S. Savasta, and F. Nori, Ultrastrong coupling between light and matter, Nat. Rev. Phys. 1, 19 (2019).

[3] P. Forn-Díaz, L. Lamata, E. Rico, J. Kono, and E. Solano, Ultrastrong coupling regimes of light-matter interaction, Rev. Mod. Phys. 91, 025005 (2019).

[4] A. Blais, A. L. Grimsmo, S. M. Girvin, and A. Wallraff, Circuit quantum electrodynamics, Rev. Mod. Phys. 93, 025005 (2021).

[5] J.-T. Shen and S. Fan, Coherent Single Photon Transport in a One-Dimensional Waveguide Coupled with Superconducting Quantum Bits, Phys. Rev. Lett. 95, 213001 (2005).

[6] J.-T. Shen and S. Fan, Strongly correlated multiparticle transport in one dimension through a quantum impurity, Phys. Rev. A 76, 062709 (2007).

[7] D. E. Chang, A. S. Sørensen, E. A. Demler, and M. D. Lukin, A single-photon transistor using nanoscale surface plasmons, Nat. Phys. 3, 807 (2007).

[8] O. Astafiev, A. M. Zagoskin, A. A. Abdumalikov, Y. A. Pashkin, T. Yamamoto, K. Inomata, Y. Nakamura, and J. S. Tsai, Resonance fluorescence of a single artificial atom, Science 327, 840 (2010).

[9] K. Le Hur, Kondo resonance of a microwave photon, Phys. Rev. B 85, 140506(R) (2012).

[10] B. Peropadre, D. Zueco, D. Porras, and J. J. García-Ripoll, Nonequilibrium and Nonperturbative Dynamics of Ultrastrong Coupling in Open Lines, Phys. Rev. Lett. 111, 243602 (2013).

[11] M. Goldstein, M. H. Devoret, M. Houzet, and L. I. Glazman, Inelastic Microwave Photon Scattering off a Quantum Impurity in a Josephson-Junction Array, Phys. Rev. Lett. 110, 017002 (2013).

[12] S. Bera, H. U. Baranger, and S. Florens, Dynamics of a qubit in a high-impedance transmission line from a bath perspective, Phys. Rev. A 93, 033847 (2016).
[13] N. Gheeraert, X. H. H. Zhang, T. Sépulcre, S. Bera, N. Roch, H. U. Baranger, and S. Florens, Particle production in ultrastrong-coupling waveguide QED, Phys. Rev. A 98, 043816 (2018).

[14] T. Shi, Y. Chang, and J. J. García-Ripoll, Ultrastrong Coupling Few-Photon Scattering Theory, Phys. Rev. Lett. 120, 153602 (2018).

[15] X. Gu, A. F. Kockum, A. Miranowicz, Y.-x. Liu, and F. Nori, Microwave photonics with superconducting quantum circuits, Phys. Rep. 718-719, 1 (2017).

[16] A. Le Boité, Theoretical methods for ultrastrong light-matter interactions, Adv. Quantum Technol. 3, 1900140 (2020).

[17] P. Forn-Díaz, J. J. García-Ripoll, B. Peropadre, J. L. Orgiazzi, M. A. Yurtalan, R. Belyansky, C. M. Wilson, and A. Lupascu, Ultrastrong coupling of a single artificial atom to an electromagnetic continuum in the nonperturbative regime, Nat. Phys. 13, 39 (2017).

[18] L. Magazzù, P. Forn-Díaz, R. Belyansky, J. L. Orgiazzi, M. A. Yurtalan, M. R. Otto, A. Lupascu, C. M. Wilson, and M. Grifoni, Probing the strongly driven spin-boson model in a superconducting quantum circuit, Nat. Commun. 9, 1403 (2018).

[19] J. Puertas Martínez, S. Léger, N. Gheeraert, R. Dassonneville, L. Planat, F. Foroughi, Y. Krupko, O. Buisson, C. Naud, W. Hasch-Guichard, S. Florens, I. Snyman, and N. Roch, A tunable Josephson platform to explore many-body quantum optics in circuit-QED, npj Quantum Inf. 5, 19 (2019).

[20] R. Kuzmin, N. Mehta, N. Grabon, R. Mencia, and V. E. Manucharyan, Superstrong coupling in circuit quantum electrodynamics, npj Quantum Inf. 5, 20 (2019).

[21] S. Léger, J. Puertas-Martínez, K. Bharadwaj, R. Dassonneville, J. Delaforce, F. Foroughi, V. Milchakov, L. Planat, O. Buisson, C. Naud, W. Hasch-Guichard, S. Florens, I. Snyman, and N. Roch, Observation of quantum many-body effects due to zero point fluctuations in superconducting circuits, Nat. Commun. 10, 5259 (2019).

[22] M. Vojta, Impurity quantum phase transitions, Philos. Mag. 86, 1807 (2006). 
[23] V. J. Emery and A. Luther, Ground-State Properties in the Kondo Problem, Phys. Rev. Lett. 26, 1547 (1971).

[24] R. Silbey and R. A. Harris, Variational calculation of the dynamics of a two level system interacting with a bath, J. Chem. Phys. 80, 2615 (1984).

[25] R. A. Harris and R. Silbey, Variational calculation of the tunneling system interacting with a heat bath. II. Dynamics of an asymmetric tunneling system, J. Chem. Phys. 83, 1069 (1985).

[26] A. J. Leggett, S. Chakravarty, A. T. Dorsey, M. P. A. Fisher, A. Garg, and W. Zwerger, Dynamics of the dissipative two-state system, Rev. Mod. Phys. 59, 1 (1987).

[27] A. Nazir, D. P. S. McCutcheon, and A. W. Chin, Ground state and dynamics of the biased dissipative two-state system: Beyond variational polaron theory, Phys. Rev. B 85, 224301 (2012).

[28] A. W. Chin, J. Prior, S. F. Huelga, and M. B. Plenio, Generalized Polaron Ansatz for the Ground State of the Sub-Ohmic Spin-Boson Model: An Analytic Theory of the Localization Transition, Phys. Rev. Lett. 107, 160601 (2011).

[29] G. Díaz-Camacho, A. Bermudez, and J. J. García-Ripoll, Dynamical polaron Ansatz: A theoretical tool for the ultrastrongcoupling regime of circuit QED, Phys. Rev. A 93, 043843 (2016).

[30] S. He, L. Duan, and Q. H. Chen, Improved Silbey-Harris polaron ansatz for the spin-boson model, Phys. Rev. B 97, 115157 (2018).

[31] E. Sánchez-Burillo, L. Martín-Moreno, J. J. García-Ripoll, and D. Zueco, Single Photons by Quenching the Vacuum, Phys. Rev. Lett. 123, 013601 (2019).

[32] V. Paulisch, T. Shi, and J. J. Garcia-Ripoll, Two-photon scattering in usc regime, arXiv:1810.08439.

[33] S. Bera, A. Nazir, A. W. Chin, H. U. Baranger, and S. Florens, Generalized multipolaron expansion for the spin-boson model: Environmental entanglement and the biased two-state system, Phys. Rev. B 90, 075110 (2014).

[34] J. Román-Roche, E. Sánchez-Burillo, and D. Zueco, Bound states in ultrastrong waveguide QED, Phys. Rev. A 102, 023702 (2020).

[35] A. H. Castro Neto, E. Novais, L. Borda, G. Zaránd, and I. Affleck, Quantum Magnetic Impurities in Magnetically Ordered Systems, Phys. Rev. Lett. 91, 096401 (2003).

[36] E. Novais, A. H. Castro Neto, L. Borda, I. Affleck, and G. Zarand, Frustration of decoherence in open quantum systems, Phys. Rev. B 72, 014417 (2005).

[37] A. M. Sengupta, Spin in a fluctuating field: The Bose(+Fermi) Kondo models, Phys. Rev. B 61, 4041 (2000).

[38] L. Zhu and Q. Si, Critical local-moment fluctuations in the Bose-Fermi Kondo model, Phys. Rev. B 66, 024426 (2002).

[39] G. Zaránd and E. Demler, Quantum phase transitions in the Bose-Fermi Kondo model, Phys. Rev. B 66, 024427 (2002).

[40] D. V. Khveshchenko, Quantum impurity models of noisy qubits, Phys. Rev. B 69, 153311 (2004).

[41] A. Baksic and C. Ciuti, Controlling Discrete and Continuous Symmetries in "Superradiant" Phase Transitions with Circuit Qed Systems, Phys. Rev. Lett. 112, 173601 (2014).
[42] S. Mahmoodian, Chiral Light-Matter Interaction beyond the Rotating-Wave Approximation, Phys. Rev. Lett. 123, 133603 (2019).

[43] J. Prior, A. W. Chin, S. F. Huelga, and M. B. Plenio, Efficient Simulation of Strong System-Environment Interactions, Phys. Rev. Lett. 105, 050404 (2010).

[44] A. W. Chin, Á. Rivas, S. F. Huelga, and M. B. Plenio, Exact mapping between system-reservoir quantum models and semiinfinite discrete chains using orthogonal polynomials, J. Math. Phys. 51, 092109 (2010).

[45] See Supplemental Material at http://link.aps.org/supplemental/ 10.1103/PhysRevResearch.3.L032058 for details on the numerical simulations, derivation of the elastic $S$ matrix and its relation to the spin susceptibilities, perturbative calculation of inelastic scattering, and a comparison with a system without frustrated couplings, which includes Refs. [58-61].

[46] E. Sanchez-Burillo, D. Zueco, J. J. Garcia-Ripoll, and L. Martin-Moreno, Scattering in the Ultrastrong Regime: Nonlinear Optics with one Photon, Phys. Rev. Lett. 113, 263604 (2014).

[47] D. C. Langreth, Friedel sum rule for Anderson's model of localized impurity states, Phys. Rev. 150, 516 (1966).

[48] G. Zaránd, L. Borda, J. von Delft, and N. Andrei, Theory of Inelastic Scattering from Magnetic Impurities, Phys. Rev. Lett. 93, 107204 (2004).

[49] L. Fritz, S. Florens, and M. Vojta, Universal crossovers and critical dynamics of quantum phase transitions: A renormalization group study of the pseudogap Kondo problem, Phys. Rev. B 74, 144410 (2006).

[50] L. Borda, L. Fritz, N. Andrei, and G. Zaránd, Theory of inelastic scattering from quantum impurities, Phys. Rev. B 75, 235112 (2007).

[51] H. Bruus and K. Flensberg, Many-body Quantum Theory in Condensed Matter Physics: An Introduction (Oxford University Press, New York, 2004).

[52] V. J. Emery and A. Luther, Low-temperature properties of the Kondo Hamiltonian, Phys. Rev. B 9, 215 (1974).

[53] P. Nozières, A "fermi-liquid" description of the Kondo problem at low temperatures, J. Low Temp. Phys. 17, 31 (1974).

[54] Y. Yao, N. Zhou, J. Prior, and Y. Zhao, Competition between diagonal and off-diagonal coupling gives rise to charge-transfer states in polymeric solar cells, Sci. Rep. 5, 14555 (2015).

[55] C. Duan, C.-Y. Hsieh, J. Liu, J. Wu, and J. Cao, Unusual transport properties with noncommutative system-bath coupling operators, J. Phys. Chem. Lett. 11, 4080 (2020).

[56] http://hpcc.umd.edu.

[57] ITensor Library (version 2.1.1) http://itensor.org.

[58] H. Suhl, Dispersion theory of the Kondo effect, Phys. Rev. 138, A515 (1965).

[59] P. Coleman, Introduction to Many-Body Physics (Cambridge University Press, Cambridge, UK, 2015).

[60] M. Srednicki, Quantum Field Theory (Cambridge University Press, Cambridge, UK, 2007).

[61] B. Bruognolo, A. Weichselbaum, C. Guo, J. von Delft, I. Schneider, and M. Vojta, Two-bath spin-boson model: Phase diagram and critical properties, Phys. Rev. B 90, 245130 (2014). 